\title{
Pierre Brunel, «Dans Venise la rouge...», in De Florence à Venise. Études en l'honneur de Christian Bec
}

\section{Annalisa Bottacin}

\section{(2) OpenEdition}

1 Journals

\section{Edizione digitale}

URL: http://journals.openedition.org/studifrancesi/7651

DOI: 10.4000/studifrancesi.7651

ISSN: 2421-5856

\section{Editore}

Rosenberg \& Sellier

\section{Edizione cartacea}

Data di pubblicazione: 1 décembre 2009

Paginazione: 652

ISSN: 0039-2944

\section{Notizia bibliografica digitale}

Annalisa Bottacin, «Pierre Brunel, «Dans Venise la rouge...», in De Florence à Venise. Études en l'honneur de Christian Bec», Studi Francesi [Online], 159 (LIII | III) | 2009, online dal 30 novembre 2015, consultato il 09 janvier 2021. URL: http://journals.openedition.org/studifrancesi/7651 ; DOI: https://doi.org/ 10.4000/studifrancesi.7651

Questo documento è stato generato automaticamente il 9 janvier 2021.

\section{(c)}

Studi Francesi è distribuita con Licenza Creative Commons Attribuzione - Non commerciale - Non opere derivate 4.0 Internazionale. 


\title{
Pierre Brunel, «Dans Venise la rouge...", in De Florence à Venise. Études en l'honneur de Christian Bec
}

\author{
Annalisa Bottacin
}

\section{NOTIZIA}

PIERRE BRUNEL, «Dans Venise la rouge....", in De Florence à Venise. Études en l'honneur de Christian Bec, réunies par François LIVI et Carlo ossoLA, P. U. Paris-Sorbonne, 2006, «Jalons», collection d'études italiennes dirigée par François Livi, nouvelle série, pp. $463-475$.

Questo rilevante contributo è contrassegnato da una riflessione intorno a un Alfred de Musset «peintre et dessinateur» prima ancora che scrittore; in effetti, l'eminente studioso evidenzia che nel 1827, una volta abbandonati gli studi di medicina, ogni mattina Musset si recava al Louvre «comme n'importe quel rapin pour copier les chefsd'œuvre des maitres» (p. 463). L'acquisizione del senso del colore e delle forme non parve comunque evidente nella sua trasmigrazione nei versi poetici a dire dei suoi contemporanei, quale ad esempio Eugène Delacroix, che ebbe a considerarlo «un poète qui n'a pas de couleur». È certo che all'epoca Musset aveva già iniziato a dedicarsi alla poesia e a leggere i grandi classici, quali Shakespeare e Schiller; Brunel rileva infatti che il suo primo incontro con Venezia «au clair de lune» avviene attraverso le pagine del Mercante di Venezia, in quanto quest'immagine si ritrova nella scena della musica al chiaro di luna della prima scena del quinto atto. Scrive Shakespeare: «How sweet the moonlight sleeps upon this bank! | Here will we sit, and let the sounds of music / Creep in our ears; soft stillness, and the night / Become the touches of sweet harmony» (p. 464).

Rimembranza certa, a dire di Brunel, trasposta nel poema Venise, la cui redazione risale quasi certamente al 1828 e che sarà pubblicato l'anno seguente nei Contes d'Espagne et d'Italie, prima di apparire nella raccolta delle Premières Poésies, di cui citiamo la prima 
strofa: «Dans Venise la rouge,|Pas un bateau qui bouge, |Pas un pêcheur dans l'eau, |Pas un falot...». Così commenta il critico, alludendo al giudizio parzialmente negativo del grande pittore: «Pourtant cette Venise de Musset n'est pas en noir et blanc. Elle ne se réduit pas à quelques traits. Elle est "Venise la rouge", et, pour une fois, la notation ferait mentir le jugement de Delacroix» (p.464). 11 poema veneziano sarà messo in musica da Charles Gounod e questa riflessione dà l'avvio a una interessante serie di esemplificazioni e accostamenti in campo musicale e poetico su temi celebranti Venezia e la luna, tra cui i Canti leopardiani. Questo rilevante gioco di coincidenze, così ben inserite una nell'altra, concorrono a rilevare con grande mae-stria da parte di Brunel, quanto verso e melodia possano combinarsi in corrispondenze segrete che ne riducono le distanze, dispiegandosi in investimenti simbolici, produttori di ineluttabili voluttà. 